\title{
EFECTOS DE LA INTEMPERIE EN EL COLOR DE DOS ACABADOS APLICADOS EN MADERA DE CEDRELA ODORATA Y CARAPA GUIANENSIS
}

\section{EFFECT OF WEATHERING ON COLOR OF TWO COATING APPLIED ON CEDRELA ODORATA AND CARAPA GUIANENSIS WOOD}

\author{
Juan Carlos Valverde ${ }^{1}$, Róger Moya ${ }^{1}$
}

\begin{abstract}
RESUMEN
Cedrela odorata y Carapa guianensis son maderas utilizadas para la elaboración de muebles, puertas e inmobiliario de casas en Costa Rica. Así mismo, dos tipos de acabados (tipo nitrocelulosa y de poliuretano) son muy utilizados en estas maderas. No obstante, los cambios en los parámetros del color (CIE L*a*b*) por el intemperismo es poco conocido para estas especies y estos acabados. Al evaluar estos acabados ante el intemperismo se encontró que el acabado con poliuretano y nitrocelulasa producen cambios en la coloración de la madera apreciables en los primeros 40-60 días de exposición a la interperie y este cambio del color fue diferente en cada acabado. Ademas, los resultados mostraron que en $C$. guianensis los cambios medido por $\Delta \mathrm{E}^{*}$ de color fueron inferiores a los obtenidos en la $C$. odorata en los dos acabados con el tiempo y que estos a su vez son inferiores si no se aplica ningún tipo de acabado. Finalmente fue encontrado que las relaciones de los parámetros de color $\left(\mathrm{L}^{*}, \mathrm{a}^{*}, \mathrm{c}^{*}\right)$ y la relación entre los mismo parámetros $\left(\mathrm{L}^{*}-\mathrm{a}^{*}, \mathrm{~L}^{*}-\mathrm{b}^{*}\right.$ y a*-b*) son alterados ante la intemperie por la aplicación de los acabados.
\end{abstract}

Palabras claves: CIE Lab, fotodegradación, UV, cambio de color, madera tropical

Carapa guianensis and Cedrela odorata wood are used for furniture and doors manufacturing in Costa Rica. Similarly, two kind wood coatings (nitrocellulose and polyurethane) are widely used in these manufactures. However, the effects of degradation in the components of color (CIE L* $\left.a^{*} b^{*}\right)$ of these coatings by the weathering is little known for these wood species and these coatings. It was evaluated these coatings under weathering and it was found that surface painted with polyurethane and nitrocellulose produced significant change in wood color during the first 40-60 days of weathering and the intensity of color change was different in two coatings. The results showed that color changes in Carapa guianensis wood were lower measured by $\Delta \mathrm{E}^{*}$ than Cedrela odorata wood in two coating of surface treatments. Finally, it was found that there are relationships among wood color parameters $\left(L^{*}, a^{*}, c^{*}\right)$ with weathering time and the relationship between color parameters $\left(L^{*}-a^{*}, L^{*}-b^{*}\right.$ and $\left.a^{*}-b^{*}\right)$ are altered by the coatings.

Key words: CIE Lab, photodegradation, UV, color change, tropical wood

\section{INTRODUCCIÓN}

La madera ha sido usada por su durabilidad natural, por ser un material sumamente versátil y con excelentes características de trabajabilidad (Peña y Rojas 2005). Sin embargo, una de las principales debilidades de este material es la susceptibilidad a la degradación del color natural por el intemperismo. La degradación del color de la madera ocurre rápidamente, en especial cuando es expuesta al intemperismo sin ningún tipo de protección o aislante (Peña y Rojas 2005). Factores ambientales como la radiación solar, la temperatura, el viento y la lluvia inciden sobre el cambio del color y se da en superficie debido a la penetración de luz ultravioleta y luz visible en la madera (George et al. 2005). La incidencia de estos tipos de luces, producen procesos químicos que se desarrollan en las paredes celulares que conforman la madera, afectando en especial a la lignina y a los extraíbles

'Instituto Tecnológico de Costa Rica, Escuela de Ingeniería Forestal, Apartado 159-7050, Cartago, Costa Rica.

Recibido: 17.05.2010. Aceptado: 30.08.2010.

Corresponding author: rmoya@itcr.ac.cr 
(Feist y Hon 1984) y que conllevan a producir carbonilo y el cromofóricos del carboxilo que son los principales compuestos orgánicos que producen cambios de color en la superficie (Deka et al. 2008, Ghost et al. 2009).

Para evitar o disminuir los procesos de degradación del color se han diseñado una gran gama de productos químicos (acabados) para aplicar sobre la superficie de la madera, los cuales disminuyen el tiempo de vida de la madera y específicamente disminuir los procesos de degradación biológica de este material (Petrič et al. 2004, Allen y Forrester 2006).

En Costa Rica la madera de cedro amargo (Cedrela odorata) y la caobilla (Carapa guianensis) son especies utilizadas para la elaboración de muebles, puertas y mobiliario en general, pero estas maderas son susceptibles a la degradación del color con las condiciones del intemperismo. Así mismo estos dos tipos de acabados son utilizados: los de tipo nitrocelulosa que consiste de compuestos de Isopropanol y los de tipo poliuretano que consisten en prepolímeros de uretano (Williams 2005a). Ambos tipos son utilizados debido a su facilidad de aplicación y su bajo costo.

Estudios llevados a cabo por Deka et al. (2008) y Schnabel et al. (2009) en especies de clima temperado establecen un modelo matemático de las variaciones del color de la madera. No obstante, este modelo se elabora no por condiciones normales de intemperismo si no por condiciones aceleradas en una cámara de control ambiental. En tanto que estudios de la variación del color de maderas tropicales por la intemperie son muy limitados (Oliveira et al. 2007, Barreto y Pastorez 2009).

Ante tal situación, el presente trabajo tiene como objetivo mostrar la variación del color, medido por el sistema CIE L*a*b* (Hunter LAB), con relación al tiempo de exposición a la intemperie de la ciudad de Cartago (Costa Rica) sobre superficies acabadas de madera de Cedrela odorata y Carapa guianensis con dos acabados: tipo poliuretano y de tipo nitrocelulosa. La información acá presentada permitirá establecer condiciones de uso de este tipo de madera con los diferentes tipos de acabado.

\section{MATERIALES Y MÉTODOS}

Características de las maderas y tipo de acabado utilizados: se utilizó madera de Cedrela odorata y Carapa guianensis. Siete tablas por especie fueron tomadas de un taller de la ciudad, la cuales presentaban un contenido de humedad de $12-14 \%$. De cada una de las tablas, se obtuvo 3 unidades experimentales $(7,21 \mathrm{~cm}$ de ancho, 20,1 mm de espesor y 15,40 cm de largo) en corte tangencial por tipo de acabado ( 3 acabados, 3 tipos de superficie y 12 muestras). Estas muestras estaban libres de daños o defectos y estaban compuestas de solo duramen. Las muestras fueron acondicionadas a un contenido de humedad de equilibrio del $12 \%$ (temperatura constante de $22{ }^{\circ} \mathrm{C}$ y una humedad relativa del 67\%). Las muestras fueron pulidas con lija \#100 y posteriormente con lija \#120, luego se procedió a la aplicación de 3 tipos de acabados (Tabla 1). La aplicación de los acabados de nitrocelulosa y poliuretano fue realizada con una pistola de tipo HVLP (High Volume Low Pressure) de rocío y una presión de tres bares.

Tabla 1. Tipos de acabados utilizados en la superficie de la madera de Cedrela odorata y Carapa guianensis.

\begin{tabular}{|l|l|}
\hline Tipo de acabado & \multicolumn{1}{c|}{ Descripción } \\
\hline Natural & La superficie se dejó en estado natural \\
\hline Nitrocelulosa & $\begin{array}{l}\text { La base del producto es de tipo metacrilato de metilo, de tipo concentrado, por } \\
\text { lo cual fue diluido en una proporción 1:3 con el diluyente. La aplicación fue } \\
\text { realizada acorde a la recomendación de la hoja técnica del producto. }\end{array}$ \\
\hline Poliuretano & $\begin{array}{l}\text { Consiste de un sellador y un acabado final de prepolímero de uretano, en este } \\
\text { caso fue utilizado sin brillo (mate). Tanto el sellador como el acabado fueron } \\
\text { aplicados siguiendo las especificaciones del fabricante. }\end{array}$ \\
\hline
\end{tabular}


Exposición de las muestras a la intemperie: fue diseñado un adictamente para colocar todas la muestras. Las muestras con los diferentes acabados fueron distribuidos aleatoriamente en el adictamente y luego fue colocado sobre el techo de una pequeña construcción, en donde no se presentaran problemas de sombra o que las muestras no recibieran las lluvias directamente. Este sitio se ubica en el campus del Instituto Tecnológico de Costa Rica, provincia de Cartago, Costa Rica.

Medición del color: las muestras fueron medidas en 5 puntos diferentes, cuatro de ellas se ubicaron a dos centímetros de los extremos y una quinta medición en el centro de la muestra. Para la determinación del color de la madera fue utilizado el espectrofotómetro marca miniSkan XE Plus de HunterLab. Se usó el sistema cromatológico estandarizado CIEL*a*b*. El rango de esta medida es de 400 a $700 \mathrm{~nm}$, con una apertura en el punto de medición de $11 \mathrm{~mm}$. Para la observación de la reflexión fue incluido el componente especular (SCI mode), en un ángulo de $10^{\circ}$, el cual es normal para la superficie del espécimen (D65/10); un campo de visión de $2^{\circ}$ (Observador estándar, CIE 1931) y un estándar de iluminación D65 (Correspondiente a Luz del día en $6500 \mathrm{~K}$ ). Las mediciones se iniciaron el 11 de marzo del 2009. Se controlaron cada 7 días hasta contabilizar un total de 63 días de exposición; posteriormente a ese período se hicieron evaluaciones cada 15 días hasta completar un total de 193 días de exposición. En dicho período del año en la ciudad de Cartago (Costa Rica) se encuentra la mitad del periodo seco con baja humedad relativa y sin precipitaciones. Luego de la segunda semana de mayo, se inicia el período de lluvias con altas humedades relativas y alta precipitaciones.

Análisis de resultados: primeramente fue establecida la diferencia entre el color de los dos acabados y el acabado natural. Este fue medido por el parámetro $\Delta \mathrm{E}^{*}$ desarrollado por Keey (2005), el cual establece que la diferencia en el color, medidos por el sistema CIE Lab, entre dos muestras es calculada por la suma de la diferencia cuadrática de cada en cada una de las coordenadas (Ecuación 1). Así mismo se calculó la diferencia de color entre el primer día y el de los otros días de medición y se realizó un análisis de regresión entre el parámetro de color y el tiempo de exposición a la intemperie. También con un análisis de regresión se estableció el grado de asociación entre $\mathrm{L}^{*} \operatorname{con} \mathrm{a}^{*}, \mathrm{~L}^{*}$ con $\mathrm{b}^{*}$ y a* con $b^{*}$. Se utilizó el programa estadístico SAS, utilizando la instrucción PROC REG.

$$
\Delta E^{*}=\sqrt{\left(\Delta L^{*}\right)^{2}+\left(\Delta a^{*}\right)^{2}+\left(\Delta b^{*}\right)^{2}}
$$

Donde: $\Delta \mathrm{L}^{*}=\Delta \mathrm{L}^{* \mathrm{M}}-\Delta \mathrm{L}^{*}{ }_{\mathrm{P},} \Delta \mathrm{a}^{*}=\Delta \mathrm{a}^{* \mathrm{M}}-\Delta \mathrm{a}_{\mathrm{P},}^{*} \mathrm{~b}^{*}=\Delta \mathrm{b}^{* \mathrm{M}}-\Delta \mathrm{b}_{\mathrm{P}, \mathrm{M}}^{*} \mathrm{M}=$ valor de coordenada de color de un tratamiento y $\mathrm{P}=$ valor de coordenada de color de otro tratamiento

\section{RESULTADOS Y ANÁLISIS}

\section{Color de la madera estado natural y efecto de los acabados en el color de la madera}

La madera de $C$. guianensis en estado natural presenta valores de 47,16 en $\mathrm{L}^{*}$, de 15,86 en $a^{*}$ y de 31,34 en b* y en la madera de $C$. odorata de 52,41, 17,19 y 30,98 respectivamente. En general en ambas especies de madera, el acabado natural obtuvieron valores positivos en los parámetros de color (Cuadro 2), por tanto el color de estas especies de madera es una combinación de tonos blancos (medido por el parámetro $\mathrm{L}^{*}$ ), rojizos (medido por $\mathrm{a}^{*}$ ) y amarrillo (medido por $\mathrm{b}^{*}$ ), mostrando con ello que el color de la madera es una composición de colores de blanco, rojo y amarrillo (Janin et al. 2001). Es importante de destacar que el color natural de la madera de $C$. guianensis y $C$. odorata presentaron de coeficientes de variación bajos, inferior a 7,02\% (Tabla 2).

El color de la madera de $C$. guianensis y $C$. odorata fue afectado por la aplicación de acabados. Cui et al. (2004) establece que los cambios en el color percebidos por el ojo humano son medidos por la variación de $\Delta \mathrm{E}^{*}$ en 4 niveles: (i) cuando $\Delta \mathrm{E}^{*}$ varía de 0 a 0.5 , el cambio de color no es catalogado como apreciable, (ii) cuando $\Delta \mathrm{E}^{*}$ se encuentra entre 1.5 a 3.0, es apenas apreciable en la superficie, (iii) cuando el rango varía de 3.0 a 6.0, el cambio es apreciable y (iv) finalmente, cuando $\Delta \mathrm{E}^{*}$ varía de 6.0 to 12.0 , el cambio de color es muy notable. Consecuentemente para este estudio y considerando estos niveles de cambio de color (Tabla 2), el acabado con poliuretano produce un 
cambio de color muy notable al ojo humano (DE* superior a 14 ), mientras que el acabado del tipo nitrocelulosa produce un cambio apreciable en la superficie de la madera tratada (DE* entre 3-6).

Tabla 2. Valores de los diferentes parámetros del color por sistema CIE L*a*b* en madera de Carapa guianensis y Cedrela odorata en diferentes tipos de superficie.

\begin{tabular}{|c|c|c|c|c|c|}
\hline \multirow{2}{*}{ Especie } & \multirow{2}{*}{$\begin{array}{l}\text { Tipo de } \\
\text { acabado }\end{array}$} & \multicolumn{3}{|c|}{ Parámetro del color } & \multirow[b]{2}{*}{$\mathrm{DE}$} \\
\hline & & $\mathrm{L}^{*}$ & $\mathrm{a}^{*}$ & $b^{*}$ & \\
\hline \multirow{3}{*}{$\begin{array}{l}\text { Carapa } \\
\text { guianensis } \\
\mathrm{N}=108\end{array}$} & Natural & $\begin{array}{c}47,16 \mathrm{~A} \\
(6,39)\end{array}$ & $\begin{array}{l}15,86 \mathrm{~A} \\
(10,25)\end{array}$ & $\begin{array}{c}31,84 \mathrm{~A} \\
(4,79)\end{array}$ & - \\
\hline & Poliuretano & $\begin{array}{c}44,99 \mathrm{~B} \\
(5,58) \\
\end{array}$ & $\begin{array}{l}14,92 \mathrm{~A} \\
(11,26) \\
\end{array}$ & $\begin{array}{c}29,85 \mathrm{~A} \\
(9,22)\end{array}$ & $\begin{array}{c}5,53 \\
(24,45) \\
\end{array}$ \\
\hline & Nitrocelulosa & $\begin{array}{c}61,19 \mathrm{C} \\
(4,26) \\
\end{array}$ & $\begin{array}{l}10,71 \mathrm{~B} \\
(10,36) \\
\end{array}$ & $\begin{array}{c}24,48 \mathrm{~B} \\
(6,30) \\
\end{array}$ & $\begin{array}{c}14,42 \\
(13,84) \\
\end{array}$ \\
\hline \multirow{3}{*}{$\begin{array}{l}\text { Cedrela } \\
\text { odorata } \\
\mathrm{N}=108\end{array}$} & $\begin{array}{l}\text { Natural } \\
\text { (testigo) }\end{array}$ & $\begin{array}{c}52,41 \mathrm{~A} \\
(3,06)\end{array}$ & $\begin{array}{c}17,19 \mathrm{~A} \\
(7,02)\end{array}$ & $\begin{array}{c}30,98 \mathrm{~A} \\
(2,76)\end{array}$ & - \\
\hline & Poliuretano & $\begin{array}{c}55,61 \mathrm{~B} \\
(2,91) \\
\end{array}$ & $\begin{array}{c}15,28 \mathrm{~B} \\
(5,82) \\
\end{array}$ & $\begin{array}{c}27,30 \mathrm{~B} \\
(4,23) \\
\end{array}$ & $\begin{array}{c}5,45 \\
(46,5) \\
\end{array}$ \\
\hline & Nitrocelulosa & $\begin{array}{c}63,95 \mathrm{C} \\
(3,25) \\
\end{array}$ & $\begin{array}{c}13,11 \mathrm{C} \\
(6,70) \\
\end{array}$ & $\begin{array}{c}23,63 C \\
(4,24) \\
\end{array}$ & $\begin{array}{c}16,95 \\
(22,17) \\
\end{array}$ \\
\hline
\end{tabular}

Nota: los valores dentro de los paréntesis corresponden a coeficiente de variación (\%) y N corresponde al número de muestras.

Los acabados transparentes para madera, como los utilizados en este estudio, en general producen un cambio en la coloración de la madera (Allen y Forrester 2006), esto porque contienen componentes estabilizadores de rayos ultravioleta, aditivos que previene el oscurecimiento (Williams 1999), pero con los cuales, en muchos casos, se hacen imposible mantener el color natural de la madera (Jewitt 2000).

\section{Variación del color con el tiempo de exposición a la intemperie}

Las variaciones de los parámetros del color $\mathrm{L}^{*}, \mathrm{a}^{*} \mathrm{y} \mathrm{b}^{*}$ en la superficie natural y en los dos tipos de acabados en las dos maderas son representados en la Figura 1. El parámetro del color L* presenta 3 fases en las dos especies: (1) aumento máximo de este valor entre el inicio de la exposición a la intemperie y los 28 y 40 días para $C$. guianensis y $C$. odorata respectivamente (Figura 1ay 1b), (2) posteriormente inicia un descenso hasta los 100 días en $C$. guianensis y 130 días en $C$. odorata y (3) luego se mantiene constante hasta el final del período de evaluación. Pero en los dos tipos de acabados el valor de L* presentó una variación con el tiempo de exposición a la intemperie diferente y en menor intensidad que la condición natural (Figura 1a). En C. guianensis inicialmente ocurre un aumento de L* entre el inicio de la exposición y los 15 días siguientes, comportamiento similar a la superficie en estado natural, pero de menor grado y en menor número de días (Figura 1a). Posteriormente decrece L* por un periodo muy corto, para luego matenerse hasta final del experimento (Figura 1a). En tanto la madera $C$. odorata presentó una disminución de $\mathrm{L}^{*}$ hasta los 60 días para mantenerse el resto de tiempo de experimento (Figura 1b). Finalmente en el acabado nitrocelulosa se presentó un comportamiento similar en ambas especies, con una disminución significativa durante los primeros 40 días de exposición, y luego un ligero aumento con el tiempo de exposición (Figura 1a y 1b). 


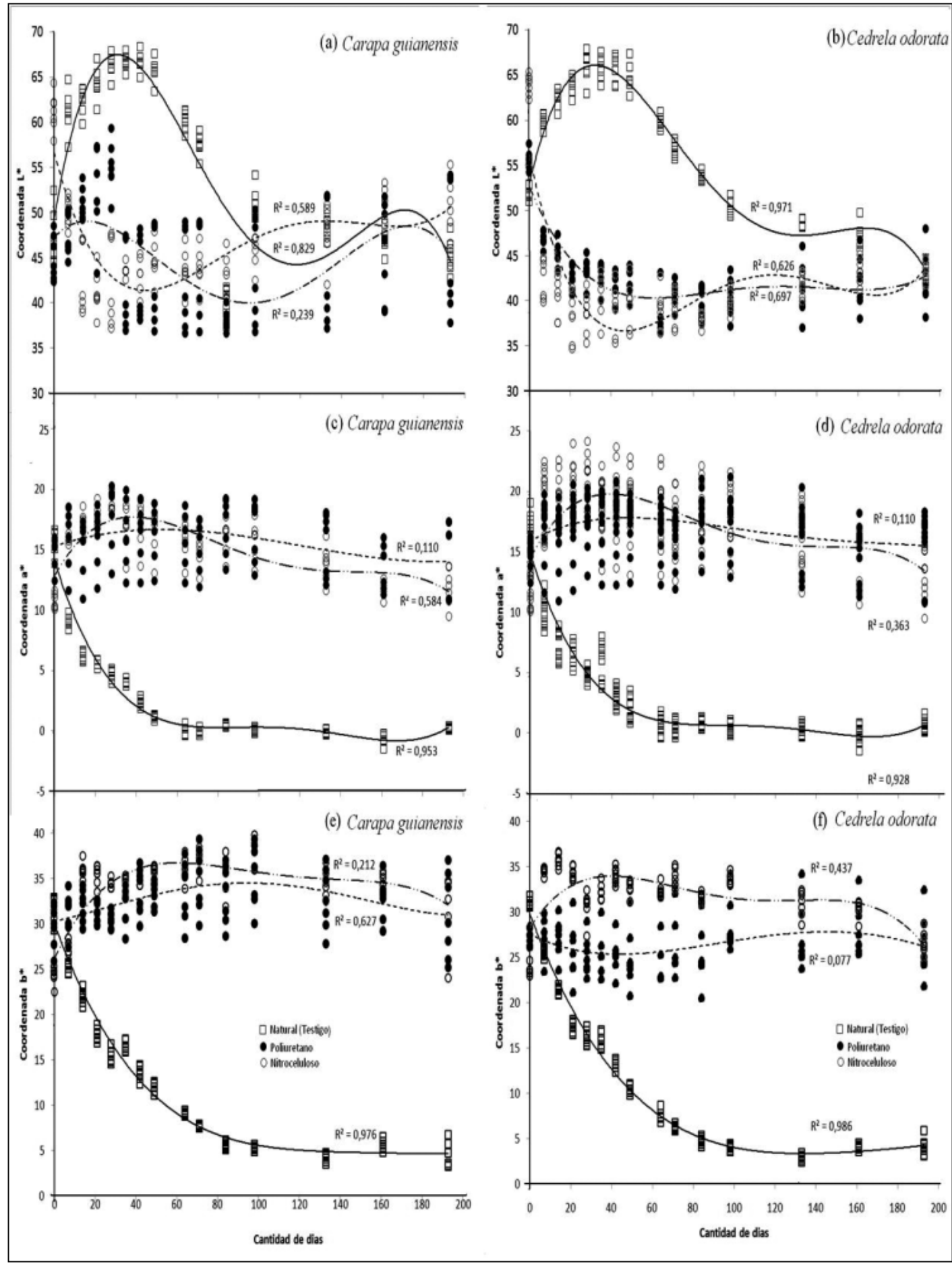

Figura 1. Variación de los parámetros del color L*, a* y b* a la intemperie de las especies Carapa guianensis y Cedrela odorata en una superficie naturaly en dos tipos de acabados.

Los parámetros de color $\mathrm{a}^{*} \mathrm{y} \mathrm{b}^{*}$ en las dos especies en estado natural presentan solamente dos fases con el tiempo a la intemperie: (1) ocurre un descenso de los valores desde el primer día exposición hasta los 61 días en el parámetro a* (Figura 1c y 1d), pero en $\mathrm{b}^{*}$, el descenso de los valores se extiende hasta el día 100 y (2) luego de este periodo se mantienen relativamente constantes (Figura 1e y 1f). El efecto de la intemperie sobre los dos tipos de acabado (poliuretano y de nitrocelulosa) se comportó de manera diferente en relación al acabado natural para estos parámetros del color. En 
el comportamiento de $\mathrm{a}^{*}$ en el acabado con poliuretano y nitrocelulosa, en ambas especies, ocurrío un ligero aumento desde el inicio de la exposición hasta los 40-50 días, para luego presentar un ligero descenso hasta el final del experimento (Figura 1c y 1d). El parametro $b^{*}$ en la superficie con poliuretano ocurre un ligero aumento en los primeros días, para luego mantenerse con el tiempo, y en el acabado con nitrocelulosa ocurre un ligero aumento entre el primer día de exposición y los 7 días, para luego mantenerse ligeramente constante (Figura 1e y 1f).

La variación del color total, medido por $\Delta \mathrm{E}^{*}$, en los dos especies, mostró que en la superficie de los 3 tratamientos ocurren cambios perceptibles (valores de $\Delta \mathrm{E}^{*}$ mayores de 6 ) del color en los primeros 40-60 días de exposición a la interperie (Figura 2) y este cambio fue diferente en los acabados de superficie. El cambio de color medido por $\Delta \mathrm{E}^{*}$ en $C$. guianensis fue inferior a $C$. odorata en los 3 tipos de tratamientos de la superficie.

El cambio del color natural de la madera comienza paulatinamente en los primeros 50 días con valores $\Delta \mathrm{E}^{*}$ que oscilan de 15 a 30 en $C$. guianensis (Figura 2a) y a los 60 días en $C$. odorata con valores de $\Delta \mathrm{E}^{*} 10$ a 30 (Figura $2 \mathrm{~b}$ ). Luego de ese incremento el valor de $\Delta \mathrm{E}^{*}$ se mantiene relativamente constante hasta el día 193 en ambas especies,. El acabado nitrocelulosa en la madera de $C$. guianensis permite cambios del color que se inician en el día 7 hasta su punto máximo en el día $35\left(\Delta \mathrm{E}^{*}\right.$ varía de 17 a 22 ) (Figura $2 \mathrm{a}$ ), en tanto que en $C$. odorata, el cambio mas fuerte ocurre a los 7 días con acabado de nitrocelulosa ( $\Delta \mathrm{E}^{*}$ varía 18 de 30 ) (Figura $2 \mathrm{~b}$ ). Luego ese punto máximo, el cambio de color de la madera se mantiente relativamente estable hasta los 100 días en ambas especies, para luego de los 100 días, mantenerse constante hasta el final del experimento (Figura 2). El acabado poliuretano, como era de esperar presentó cambios de color inferiores al acabado con nitrocelulosa, y desde luego, menor al acabado natural (Figura 2). En la madera de C. guianensis y C. odorata el máximo cambio de color se presentó en los primeros 20 días $\left(\Delta \mathrm{E}^{*}\right.$ varío de 5 a 10 en $C$. guianensis y de 10 a 15 en $C$. odorata). Posteriormente el color se mantiene relactivamente constante hasta el final del experimento (Figura 2). Es de notar que el acabado con poliuretano en $C$. odorata varío en mayor intensidad que la madera de $C$. guianensis (Figura 2). Por consiguiente, la mejor combinación de especie y acabado es la madera $C$. guianensis con poliuretano.

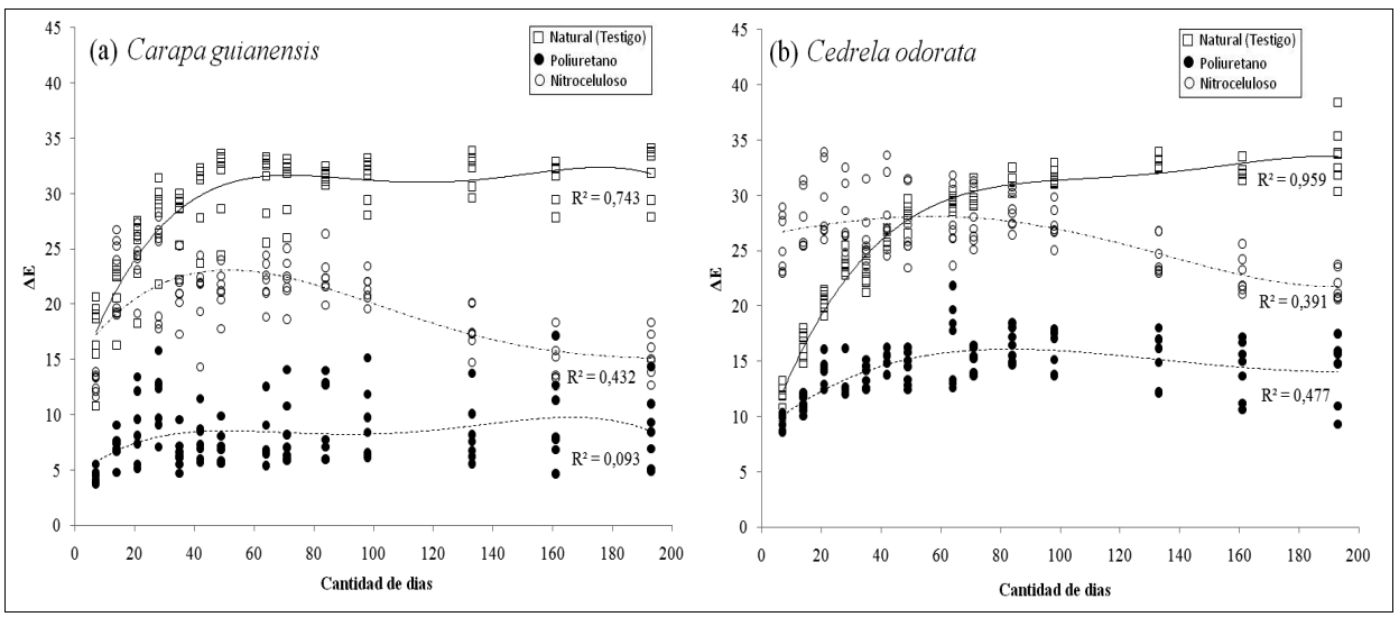

Figura 2. Comportamiento del $\Delta \mathrm{E}^{*}$ en la madera de Carapa guianensis y Cedrela odorata para 3 tipos de acabados frente a la intemperie.

Durante la exposición a la intemperie, la madera sufre una degradación atribuida a la fotodegradación de los componentes de la pared celular (celulosa y lignina) por la incidencia de los rayos UV de la luz solar (Ghost et al. 2009), sin embargo esto no ocurre luego de varios días (Williams 2005b). Durante los primeros días, los extractivos que contiene alógenos, sulfuros y nitrógenos son fácilmente degradados a baja energía contenida en los rayos UV (Williams 2005a). Esta degradación se produce probablemente durante los primeros días en la madera de C. guianensis y $C$. odorata, dando como resultado una pérdida su tonalidad, por tanto tornándose ligeramente más 
blanca, aumento de $\mathrm{L}^{*}$ a los 28 y 40 días para $C$. guianensis y $C$. odorata respectivamente (Figura 1a y $2 b)$.

El punto máximo en el valor de L* (Figura 1 b y $1 \mathrm{c}$ ), coincide con el inicio del período de lluvias en el sitio donde fueron expuestas las muestras. Esta situación puede ser explicada por el hecho que los componentes generados de la degradación de los extractivos por bajas energía de los rayos UV, son lavados por la presencia de la lluvia en la superficie de la madera (Hon, 1973), dejando una superficie emblanquecida. Así mismo esta situación da lugar a que la celulosa quede expuesta a la degradación biológica. La lignina es el primer componente que se empieza a degradar al exponer la madera (Ghost et al. 2009), esto porque la lignina absorbe la mayor cantidad de energía de los rayos UV (80-90), seguido de la holocelulosa (5-20\%) y otros extractivos de alto peso molecular (Norrström 1969). La lignina empieza su fotodegradación por la oxidación de los polímeros aromáticos (Lin y Gierer 1972), resultando en la formación de cromóforos para luego reducir a la formación de carbonilos y componentes con grupos carboxilos (Lin y Kringstad 1970). Estos nuevos grupos formados son los que le producen a la madera el color gris (Dirckx et al. 1992). Esta situación da como resultado que la madera empiece a disminuir su componente L* (Figura 3a y 3b). En tanto que la disminución de los valores de color $\mathrm{a}^{*}$ y b* (Figura 1a-1e). Algunos autores (Kamdem y Grelier 2002, Ayadi et al. 2003) lo atribuyen a la formación de quinonas y quinamos debido a la despolimerización y oxidación de la lignina. Estos cambios en coloración dan como resultado el color gris típico de la madera expuesta a la intemperie.

Los anteriores resultados muestran que la presencia del acabado disminuye el efecto de la intemperie en el color de la madera. En el caso de superficies acabadas, la radiación UV degrada primeramente los componentes químicos del acabado y luego inicia el proceso de degradación de la superficie de la madera (Creemers et al. 2005), permitiendo de esta manera mantener durante más tiempo el color original de la madera.

\section{Relación de parámetros de colores en los diferentes tipos de acabados frente a la intemperie}

La relación entre los parámetros del color durante la exposición a la intemperie variaron, no sólo para el tipo de acabado de la superficie, si no que también para las dos especies de madera. En las dos especies durante la exposición a la intemperie de la superficie en estado natural, se presentó una relación estadisticamente significante entre $\mathrm{L}^{*} \operatorname{con} \mathrm{a}^{*}, \mathrm{~L}^{*} \operatorname{con} \mathrm{b}^{*} \mathrm{y} \mathrm{a}^{*}$ con $\mathrm{b}^{*}$ (Figura 3). No obstante, la tendencia en cada una de las especies varía. En C. guianensis la relación de $\mathrm{L}^{*}$ con $a^{*}$ es positiva (Figura 3a), pero en la especie $C$. odorata el comportamiento es difrente, durante la exposición aumentó el valor de $\mathrm{L}^{*} \mathrm{y}$ disminuyó a* (Figura $3 \mathrm{~b}$ ). La relación entre los parametros de color de $\mathrm{L}^{*} \mathrm{y} \mathrm{b*}$, al igual como sucede en la relación $\mathrm{L}^{*}$ con $\mathrm{a}^{*}$, varío entre especie (Figura $5 \mathrm{c}$ y $5 \mathrm{~d})$. Durante la exposición de la superfiecie de la madera en estado natural el incremento de valor $\mathrm{L}^{*}$ produce un aumento de $\mathrm{b}^{*}$ en $C$. guianensis (Figura 3c), pero en el caso de C. odorata este aumento de $\mathrm{L}^{*}$ produce una disminución de $\mathrm{b}^{*}$ (Figura $3 \mathrm{~d}$ ). Finalmente, la relación encontrada para $\mathrm{a}^{*}$ con $\mathrm{b}^{*}$ fue positiva y estadisticamente significante en las dos especies, un incremento de $\mathrm{a}^{*}$ produce un incremento de $b^{*}$ (Figura 3e y $3 f$ ).

Las relaciones encontradas entre los diferentes parámetros del color durante la exposición a la intemperie para los dos tipos de acabados es diferente a las relaciones que se producen cuando en la madera no se aplica ningún tipo de acabado. La relación entre $\mathrm{L}^{*}$ con $\mathrm{a}^{*}$ fue negativa, sin embargo en C. guianensis fue estadisticamente significativa en los dos acabados (Figura 3a), lo que no sucedió con C. adorata, donde la relación no fue significativa (Figura 3b). Estas relaciones de los parámetros del color en los acabados difiere de las relaciones encontradas en la superficie sin ningún tipo de acabado, donde fue positiva en $C$. guianensis y negativa en $C$. odorata (Figura 3a y $3 \mathrm{~b}$ ). Las relaciones de $L^{*}$ con $b^{*}$ no fueron significantes para las dos especies de madera, contrario a lo que sucede en las superficie de color natural donde si fueron estadisticamente significantes (Figura 3c y 3d). Para la relación de $\mathrm{a}^{*}$ con $\mathrm{b}^{*}$ se encontró que esta no fue significante en dos tipos de acabados en C. guianensis (Figura 3g), pero en C. odorata la relación fue positiva y significante en el acabado de nitrocelulosa y no significante en el acabado de poliuretano, siendo esta última relación similar a la relación encontrada en la superficie de color natural (Figura 3f). 
Los anteriores resultados muestran que las variaciones de un componente del color en madera acabada son diferentes a las variaciones de ese mismo componente en madera con acabado natural, por lo que es de esperar que la intemperie actúe de diferente forma en los componentes del color en una superficie acabada.
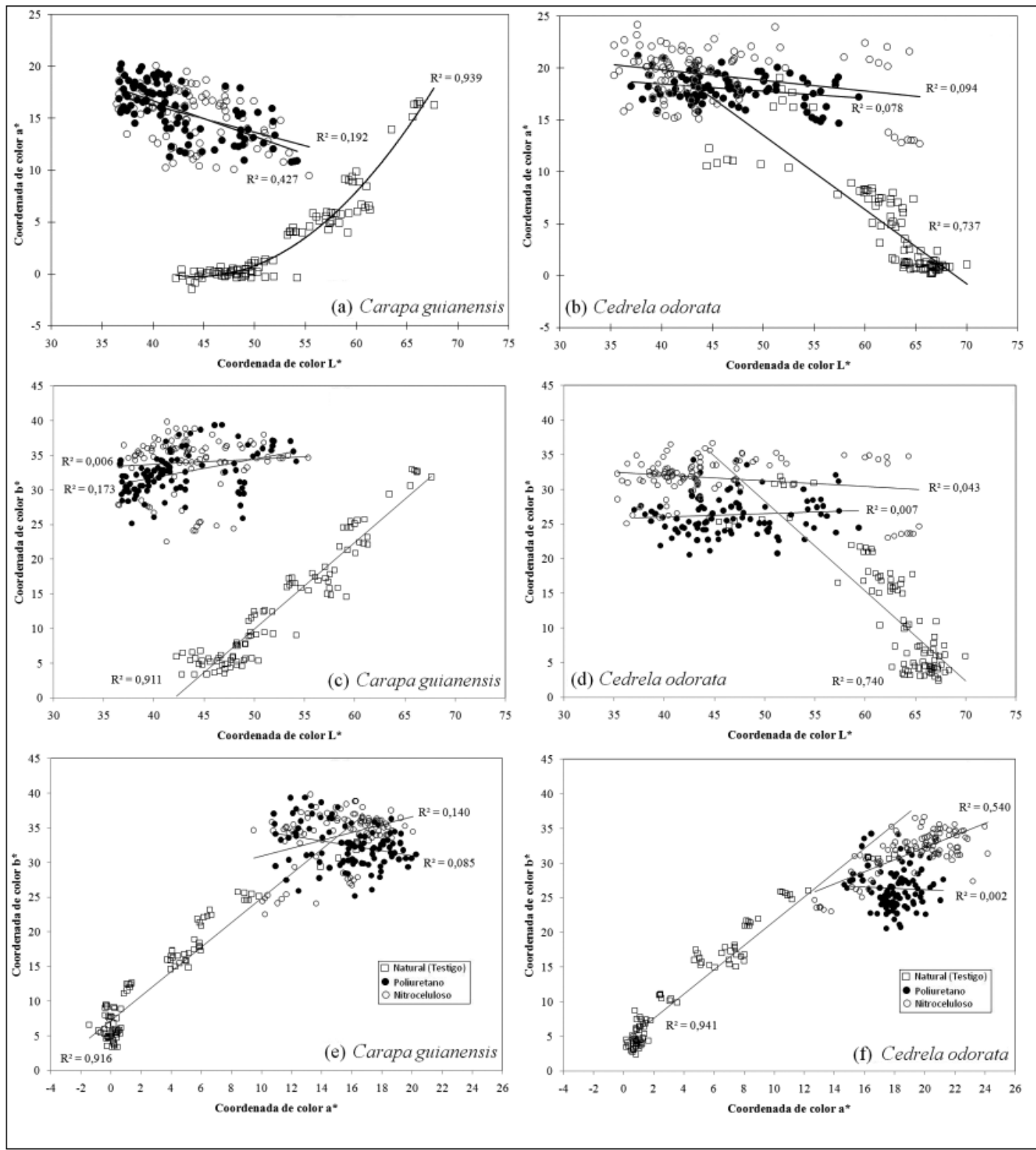

Figura 3. Relaciones de los parámetros del color L*, a* y b* de madera de Carapa guianensis y Cedrela odorata para 3 tipos de superficie frente a la intemperie.

\section{CONCLUSIONES}

La función principal de los acabados es la protección ante la intemperie, no obstante esta protección depende del tipo de acabado. Aunque el color de la madera es más estable cuando la superficie está protegida por un acabado tipo nitrocelulosa o de tipo poliuretano estos no llega a dar una protección total, ya que el cambio de color, aunque de menor grado, ocurre en los dos tipos de acabados. Con el acabado de nitrocelulosa el color cambia más rápido y con mayor intensidad del que ocurre en la superficie con acabado de tipo poliuretano en las dos especies de madera. El cambio de color se empieza a percibir luego del día 40-60 $\left(\Delta \mathrm{E}^{*}\right)$ en poliuretano y en 20-30 días en el acabado con nitrocelulosa, por lo que dichos acabados no satisfacen el desempeño frente a 
las condiciones de intemperie. Acorde con los resultados, en caso que se utilicen estos acabados en madera es necesario dar luego del día 100 un mantenimiento del acababo ya que el color empieza a cambiar. El acabado de poliuretano presenta un mejor desempeño, no obstante este también no esta eximido de un mantenimiento de la superficie. Acorde con los resultados del cambio de color $\Delta \mathrm{E}^{*}$ en los 193 días este se ha mantenido ligeramente constante, indicando con ello que aún no es necesario dar el acabado a la superficie de la madera de las dos especies.

Los resultados de las relaciones entre los diferentes parámetros de color frente a la intemperie muestran que estas fueron alteradas por la presencia del acabado, en relación con al color natural, indicando que las variaciones del color producidos en el acabado son producto de la degradación del acabado y no la degradación que ocurre en los componentes químicos de la madera cuando no le es aplicado ningún tipo de acabado.

\section{AGRADECIMIENTOS}

A la Vicerrectoría de Investigación y Extensión del Instituto Tecnológico de Costa Rica por el apoyo financiero al Proyecto de Investigación. Agradecemos además el aporte científico de los examinadores de este artículo.

\section{REFERENCIAS}

Aguilar, D.; Moya, R.; Tenorio, C. 2009. Wood color variation in undried and kiln-dried plantationgrown lumber of Vochysia guatemalensis. Madera. Ciencia y tecnología 11(3): 207-216

Allen, M.; Forrester, P. 2006. The Complete Guide to Wood Finishes: How to Apply and Restore Lacquers, Polishes, Stains and Varnishes. Fireside Books, New York, USA.

Ayadi, N.; Lejeune, F.; Charrier. F.; Charrier, B.; Merlin, A. 2003. Colour stability of heat treated wood during artificial weathering. Holz als Roh und Werkstoff 61:221-226

Barreto, C.; Pastorez, T. 2009. Resistance to artificial weathering of four tropical woods: The effect of the extractives. Ciencias Forestais 19(1): 23-30

Creemers; J.; De Meijer, M., Zimmermann, T.; Sell, J. 2002. Influence of climatic factors on the weathering of coated wood. Holz als Roh- und Werkstoff 60 (2002) 411-420

Cui, W.; Kamdem, P.; Rypstra, T. 2004. Diffuse reflectance infrared fourier transform spectroscopy (DRIFT) and color changes of artificial weathered wood. Wood Fiber Science 36: 291-301

Dirckx, O.; Triboulot-Trouy, M.C.; Merlin, A.; Deglise, X. 1992. Modifications de la couleur du bois d’Abies grandis expore a la lumiére solaire. Annals Forests Science 49: 425-447

Deka, M.; Humar, M.; Kricej, G.B., Petric, M. 2008. Effects of UV light irradiation on colour stability of thermally modified, copper ethanolamine treated and non-modified wood: EPR and DRIFT spectroscopic studies. Wood Science Technology 42:5-20

Feist, W.C.; Hon, D.N.S, 1984. Chemistry of Weathering and Protection. In: Rowell, RM (ed.) The Chemistry of Solid Wood, Advances in Chemistry Series No. 207, Chapter 11, pp. 401-451. American Chemical Society, Washington, USA.

George, B; Suttie, E; Merlin, A; Deglise, X. 2005. Photodegradation and photostablisation of woodThe state of the art. Polymer Degradation Stability 88: 268-274

Ghost, S.C.; Militz, H.; Mai; C. 2009. Natural weathering of scots pine (Pinus sylvestris L.) boards modified with functionalized commercial silicone emulsions. Bioresources 4(2): 659-673 
Hon, D.N.S. 1973. Formation of free radicals in photoirradiated cellulose and related compounds. Journal Polymer Science Chemistry Education 13: 955

Kamdem, D.P.; Grelier, S. 2002. Surface roughness and colour change of copper amine and UV absorbertreated red maple (Acer rubrum) exposed to artificial ultraviolet light. Holzforschung 56: $473-478$

Keey, R. 2005. Colour development on drying. Maderas. Ciencia y tecnología 7: 3-16

Janin, G.; González, J.; Ananias, R.; Charrier, B.; Fernandes, G.; Dilem, A. 2001. Aesthetics appreciation of wood colour and patterns by colorimetry. Part 1. Colorimetry theory for the CIELab Systems. Maderas. Ciencia y tecnología 3: 3-13

Jewitt, J. 2000. Great wood finishes. Taunton Press, USA.

Lin, S.Y.; Gierer, J. 1972. Photodegradation of lignin. A contribution to the mechanism of chromophore formation. Svensk Papperstidn 75: 233-239

Lin, S.Y.; Kringstad, K.P. 1970. Photosensitive groups in lignin and lignin model compounds. Tappi 53: $658-663$

Moya, R.; Berrocal, A., 2010. Wood colour variation in sapwood and heartwood of young trees of Tectona grandis and its relationship with plantation characteristics, site, and decay resistance. Annals Forest Science 67(1) : 109-118.

Norrström, H. 1969. Light absorbing properties of pulp and pulp components. Svensk Papperstidn $72: 25-38$

Oliveira, J; Monteiro, T; Pastore, F. 2007. Resistência ao intemperismo artificial de quatro madeiras tropicais: o efeito dos extrativos. Ciencias Forestales 17 (1):17-23

Pâques, L.E. 2004. Colour of larch heartwood and relationships to extractives and brown-rot decay resistance. Trees: Structure and function 18: 102-108

Peña, V.; Rojas, M. 2005. Tecnologías de la madera. 3 ed. Madrid, ES. Ediciones Mundi-Prensa. Madrid.

Petrič, M.; Kricej, B.; Humar, H.; Pavlic, M.; Tomazic, M. 2004. Pagination of cherry wood and spruce wood with ethanolamine and surface finishes. Surface Coatings International Part B: Coatings Transactions 87(B3):95-201

Schnabel, T; Zimmer, B; Petutschnigg, A. 2009. On the modeling of colour changes of Wood surfaces. Wood products 67: 141-149

Williams, S. 1999. Finishing of Wood. In: Wood Handbook: Wood as an engineering material. Forest Products Laboratory General Technical Report FPL-GTR-113. Forest Products Laboratory, Madison, WI. USA.

Williams, S. 2005a. Finishing of Wood. In: Willians, S. Wood handbook In Handbook of Wood Chemistry and Wood Composites. CRC press, USA.

Williams, S. 2005b. Weathering of Wood. In: Willians, S. Handbook of Wood Chemistry and Wood Composites. CRC press USA. 